Check for updates

Cite this: RSC Adv., 2017, 7, 25892

Received 23rd March 2017

Accepted 7th May 2017

DOI: $10.1039 / \mathrm{c} 7 \mathrm{ra03395h}$

rsc.li/rsc-advances

\section{Multifunctional hard-shelled microbubbles for differentiating imaging, cavitation and drug release by ultrasound $\uparrow$}

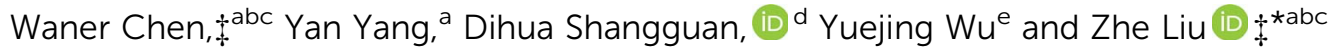

Polymeric microbubbles bearing a hard shell exhibit prominent stability and tunable acoustical properties that serve the purposes of biomedical imaging and ultrasound (US)-triggered cavitations. It is of great significance to expand the utility scope of hard-shelled microbubbles with multifunctionality, which will dramatically enhance the efficiency and precision of disease-oriented treatments. To this end, the multifunctional hard-shelled microbubbles (PMBs) for US imaging and US-triggered stimuli-responsive cavitations have been synthesized via a one-step in situ polymerization. Varied parameters including US frequency, acoustical powers and pulse duration time have been screened to optimize the cavitation conditions. It was notable to observe that by use of PMBs, a US-triggered progress of imaging, stable and inertial cavitations could be easily differentiated with an elaborately modulated parameter, which gives a visualizable pathway for imaging, stimuli-responsive cavitation, drug transportation and release at each stage. Meanwhile, commercial US contrast agents (Sonovue and Xueruixin with lipid and protein shell materials) have been compared with PMBs in terms of their cavitation performances. These valuable findings imply a promising perspective to use these multifunctional microbubbles as a novel visualizable theranostic strategy against diseases.

\section{Introduction}

The development of microbubbles (MBs) has opened up a new era for biomarker-targeted diagnostic imaging, drug delivery and ultrasound (US)-mediated therapy meeting the requirements of precision and individualized medicine. ${ }^{1-5}$ As both contrast agents and drug carriers, MBs can load therapeutic payloads and transport them to diseased lesions for USmediated theranostics. As gas-filled colloidal materials, they generally consist of an inert gas core and a shell composition of lipid, protein or polymer with a typical diameter of $0.5-10 \mu \mathrm{m} .{ }^{6,7}$ Due to dramatic acoustical impedance mismatch between MBs

${ }^{a}$ Department of Ultrasonic Diagnosis, The Second Affiliated Hospital and Yuying Children's Hospital, Wenzhou Medical University, Wenzhou 325027, Zhejiang, China.E-mail: liuzhe@wibe.ac.cn

${ }^{b}$ Wenzhou Institute of Biomaterials and Engineering, Wenzhou Medical University, Wenzhou 325001, Zhejiang, China

'Wenzhou Institute of Biomaterials and Engineering, Chinese Academy of Sciences, Wenzhou 325011, Zhejiang, China

${ }^{d}$ Beijing National Laboratory for Molecular Sciences, Key Laboratory of Analytical Chemistry for Living Biosystems, CAS Research/Education Center for Excellence in Molecular Sciences, Institute of Chemistry, Chinese Academy of Sciences, Beijing 100190, China

${ }^{e}$ Tianjin First Center Hospital, Tianjin 300192, China

$\dagger$ Electronic supplementary information (ESI) available: Experimental details including fabrication and characterization of polymeric microbubbles, and the screening for optimized US cavitation parameters. See DOI: 10.1039/c7ra03395h $\ddagger$ These authors contributed equally to this work. and tissues, signal enhancement can be generated and therefore contrast-enhanced US (CE US) images can be reconstructed for convenient visualization and recognition. In particular, USinduced inertial or stable cavitations at the presence of MBs have attracted roaring attention in the fields of cancer-oriented therapy and micro-invasive surgery. ${ }^{\mathbf{8} 9}$ In consequence, the fabrication of novel MBs with prominent physical and acoustical properties is a key prerequisite in this regard.

According to different shell materials, soft-shelled (lipidbased) and hard-shelled (polymeric and protein-based) MBs can be sorted. ${ }^{10}$ Although soft-shelled MBs are broadly used in clinics as injectable contrast agents for cardiovascular perfusion imaging, hard-shelled MBs have shown apparent advantages such as higher in vivo stability, relatively thicker shell for enhanced drug-loading, better tolerance for destructive imaging and controlled drug release, and easier access to chemical modification for bio-targeting. ${ }^{\mathbf{1 0 , 1 1}}$ On the other hand, it is well known that exposure of ultrasound to MBs at different mechanical indexes (MI) gives birth to either stable (steady oscillation) or inertial (rapid growth into collapse) cavitations. ${ }^{12,13}$ The resonance oscillation and collapse of drugentrapped MBs simultaneously induces the enhanced vascular permeability, which has become an effective route to successful treatments of various diseases. ${ }^{\mathbf{1 4}}$ To envisage the theranostic efficacy, choices of shell materials is vitally important since they predominantly determine the acoustical profiles and the extent to which MBs can oscillate during US irradiation. ${ }^{15}$ Hard-shelled MBs demonstrate more US sensitivity especially at high MI, 


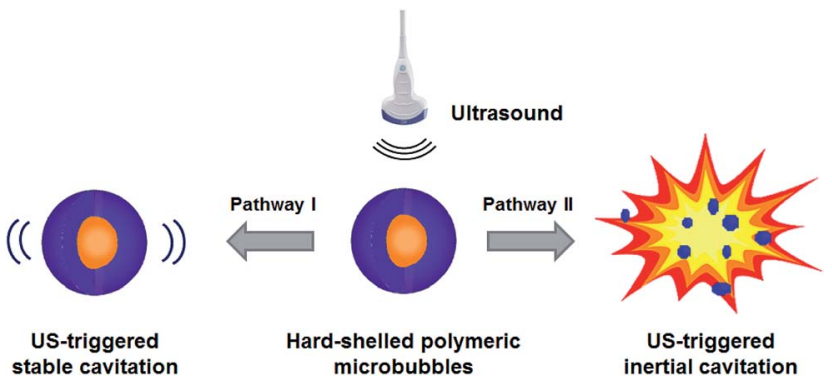

Scheme 1 Optimization for US-triggered stable and inertial cavitations by using hard-shelled polymeric microbubbles (PMBs) as multifunctional agents.

which ensures them suitable for destructive color doppler US or US-triggered stimuli-responsive drug release. ${ }^{16,17}$ To sum up, appropriate shell materials and optimized cavitation conditions are playing an important role in the US-triggered theranostic strategy for final successful treatments in the case of either clinical or pre-clinical applications.

Polymeric MBs as representative hard-shelled US contrast agents, have recently gained increasingly interest due to their non-immunogenicity and easy fabrication protocols. Poly $n$ butyl cyanoacrylate (PBCA) is a biocompatible and biodegradable material that has been extensively utilized in clinics with sufficient bio-safety. Thus, the use of PBCA as the shell material to construct MBs will pave the way to clinical translation. Our previous studies have reported the use of PBCA-based MBs for US imaging. ${ }^{17-20}$ As a follow-up investigation, we herein describe the PBCA-based polymeric microbubbles (PMBs) and their optimization for US-triggered stable and inertial cavitations as a potential strategy for drug transportation and stimuliresponsive release (Scheme 1). Based on this concept, we synthesized these polymeric MBs and screened varied parameters such as US frequency, acoustical power, duty cycles, mechanical index, pulse duration time to study the oscillation performance and cavitation effect as well as their optimized resonance conditions. Meanwhile, these profiles of assynthesized PMBs have been compared with the commercial US MBs, Sonovue and Xueruixin, to disclose their outstanding acoustical properties. To the best of our knowledge, it is the first report so far to grope the optimized conditions for US-triggered cavitations of the hard-shelled PMBs with clinical ultrasound devices. These findings will not only provide solid evidences to PMB-related theranostic applications which combine imaging, diagnosis and therapy in one single process with higher doseeconomy and bio-availability, but also shed bright perspectives to image-guided therapy or image-navigated surgery, so that a synergistic treatment with higher precision and efficiency in combination of US and PMBs will be realized.

\section{Results and discussions}

\subsection{Synthesis and characterization of hard-shelled polymeric microbubbles (PMBs)}

The hard-shelled polymeric microbubbles (PMBs) were prepared via a one-step emulsion polymerization of butyl cyanoacrylate
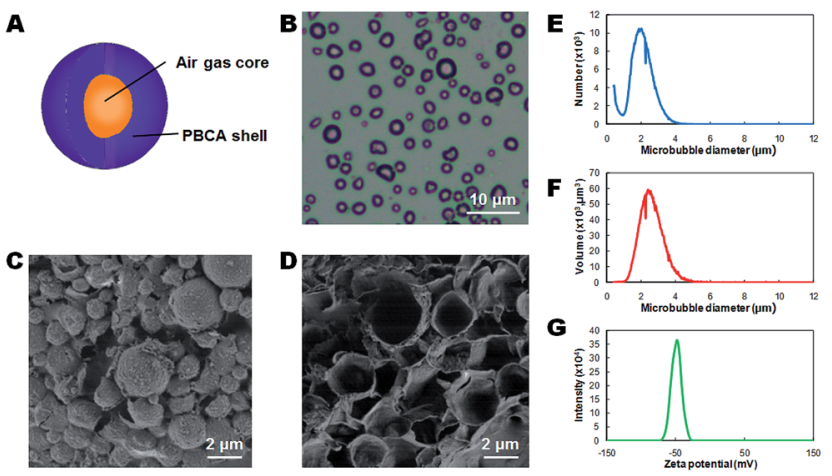

Fig. 1 Illustration of hard-shelled polymeric microbubbles (PMBs) (A) and characterization by optical microscopy (B), scanning electron microscopy (SEM) (C and D), number-dependent and volume-dependent size distribution (E and F), and zeta potential measurements (G).

(BCA) as monomers. In this facile synthetic procedure, air was incorporated as the gas core, and the shell was formed along with BCA polymerization during the high-speed agitation. Triton X-100 as a neutral surfactant contributed to the stabilization of PMBs. The polymerization was complete in $4 \mathrm{~h}$ with a following centrifugation step as the size-isolation procedure to harvest the monodispersed MBs. The as-synthesized PMBs exhibited a typical coreshell structure as shown schematically in Fig. 1A. The optical microscopy image indicated that PMBs had excellent stability and a narrow size distribution without obvious aggregation (Fig. 1B). The SEM image clearly demonstrated the surface morphology of PMBs and also provided a pathway to estimate their sizes. They showed a spherical shape with a relatively rough surface but evenly distributed (Fig. 1C). Moreover, the SEM image of disintegrated PMBs probably due to SEM vacuum atmosphere indicated that the polymeric shell thickness was approximately $50 \mathrm{~nm}$, and the air gas core was also validated (Fig. 1D). To further study the size and volume distribution of PMBs, coulter counter measurements were applied and the results collectively evidenced their uniform distribution with an average size of $2.13 \pm 0.55 \mu \mathrm{m}$ either for number-dependent or volume-dependent measurements (Fig. 1E and F). The in situ polymerization of BCA also endowed PMBs with negative surface charges with a zeta potential of $-49.0 \pm 6.2 \mathrm{mV}$ (Fig. 1G), which provided their superb stability and possibility for further surface modifications e.g. biomarkertargeted ligand conjugation. These features not only give full access to the application of PMBs as US contrast agents and carriers for imaging and drug loading, but also encourage us to make further investigations on US-triggered cavitations at the presence of PMBs.

\subsection{Screening of multiple parameters for optimized cavitation effects at the presence of PMBs}

It is highly desired to apply these polymeric microbubbles to multifunctional theranostic purposes. By either stable or inertial cavitations, they will play multiple roles as US contrast agents for imaging, cavitation agents and payload carriers for US-triggered drug transportation and release. To this end, it is prerequisite to explore the cavitation parameters such as US 
frequency, acoustical power, duty cycles, mechanical index and pulse duration time. Inertial cavitation will give rise to the "bubble bombing", and simultaneously physical effects such as micro-streaming, shockwaves, local shear force and micro-jets are generated which significantly contribute to the increased trans-cellular drug uptake as a result of reversible structural deformations on cell membranes. ${ }^{21,22}$ In comparison, stable cavitation will not lead to the destruction of MBs but is conducive to US imaging. Hence, it is valuable to optimize the critical conditions under which strongest bubble resonance will give birth to enhanced drug release at the targeted lesions at no sacrifice of US imaging efficiency. ${ }^{23,24}$

2.2.1 Ultrasound frequency and acoustical power. Studies have shown that cavitation effect is closely related to the US MI, and cavitation is unlikely to occur with an MI value less than 0.7. Nevertheless, the cavitation threshold might be substantially reduced when MBs are utilized as efficient cavitation agents. ${ }^{25-27}$ To explore the optimized cavitation parameters, US frequency and acoustical power, two factors which greatly influence the MI values, were firstly selected via bi-variant experiments. PMBs were immobilized in gelatin as the phantom with a final concentration of 1500 MBs per $\mu \mathrm{L}$, and a US transducer was placed over the phantom to pose the ultrasound and record the images by using a color doppler imaging system (Fig. 2A). The duty cycles were calculated by eqn (1), and it was noticed that when frequency was adjusted to 1.7 $\mathrm{MHz}$ and 2.0 $\mathrm{MHz}$, the pulse length was set to an optional maximum of 17.5 and 21 cycle respectively. ${ }^{28-30}$ All the duty cycles with varied frequency and pulse length were rather small as shown in Table $\mathbf{S 1 , \dagger}$ and the MI values in variation with US frequencies and acoustical powers have been listed in Table S2. $\dagger$

The US images at varied frequencies and acoustical powers were recorded as Fig. S1-S9. $\dagger$ It is obviously demonstrated that with acoustical power increased, signal intensity post-US irradiation decreased sharply due to the collapse of PMBs. Therefore, a palpable rise of \%decrease that varied with the enhanced acoustical powers indicated the cavitation effect gradiently transited from stable to inertial (Fig. $2 \mathrm{~B}$ and C). ${ }^{17}$ The darkness
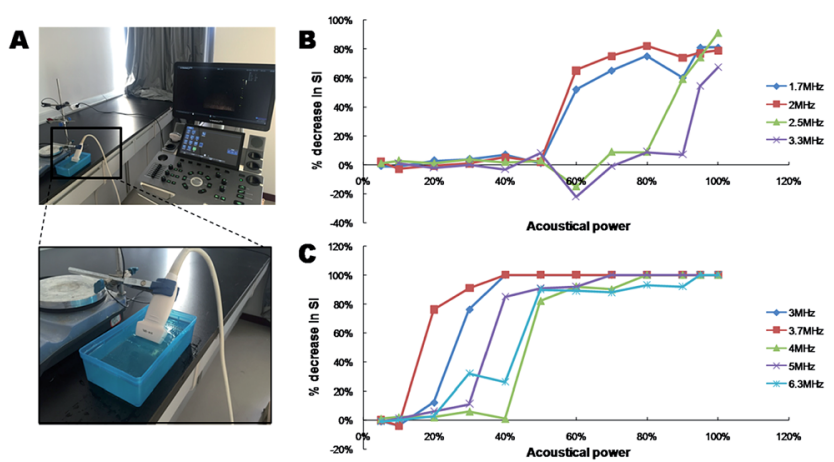

Fig. 2 The experimental apparatus (A) and cavitation effects by evaluating \%decrease of US signal intensity (SI) of pre- and post-US irradiation images at varied US frequencies and acoustical powers by using a convex transducer S1-8C (B) and a linear transducer X4-12L (C) respectively. in region of interest (ROI) of post-cavitation images was almost $100 \%$ SI decrease which was ascribed to an inertial cavitation at the presence of PMBs. In contrast, US signals in ROI at lower acoustical powers exhibited slightly decreased or intact, which implied a stable or partial inertial cavitation. It is interestingly to note a negative data-point of \%decrease $(-15 \%(2.5 \mathrm{MHz}$, $60 \%)$ and $-22 \%(3.3 \mathrm{MHz}, 60 \%))$ as a result of an obvious signal enhancement in ROI, which implied the occurrence of stable cavitation at the critical resonance frequency of PMBs (Fig. 2B, $\mathrm{S} 3$ and $\mathrm{S} 4 \dagger) .{ }^{31}$ As for cavitations at lower frequencies $(1.7,2.0$, 2.5 and $3.3 \mathrm{MHz}$ ) by using a convex transducer (S1-8C), no obvious inertial cavitations could be observed when the acoustical power was lower than $50 \%$. With regard to higher frequencies $(3.0,3.7,4.0,5.0$ and $6.3 \mathrm{MHz}$ ) by using a linear transducer (X4-12L), inertial cavitations could be achieved with the acoustical power set lower than $50 \%$, while no inertial cavitation was detected with the acoustical power lower than $10 \%$. Hence, these findings clearly evidenced that the cavitation were activated by ultrasound at the presence of PMBs, and the appropriate selection of frequency and acoustical power influenced the cavitation effects accordingly. Considering that the resonance frequency of microbubbles could be reduced by modulating their sizes and shell stiffness, ${ }^{32-34}$ the presence of PMBs in this case have lowered the inertial cavitation MI threshold dramatically to nearly 0.3 (Table $\mathrm{S} 2 \dagger$ ).

2.2.2 US pulse duration time. The noteworthy phenomenon that US irradiation at a specific frequency of $3.3 \mathrm{MHz}$ and acoustical power of $60 \%$ for $12 \mathrm{~s}$ led to a prominent SI enhancement of $22 \%$ reminded us to take pulse duration time into consideration as a key factor that might affect the optimized cavitation effect. Under the defined irradiation conditions (frequency: $3.3 \mathrm{MHz}$, acoustical power: $60 \%$ ), the PMBs phantom was treated with US pulses irradiating the same ROI for a total duration time of $44 \mathrm{~s}$. The pulse paused every $2 \mathrm{~s}$ and US images were taken for SI analysis all through the imaging sequence (Fig. 3 and S10 $\dagger$ ). As shown, the brightness in ROI gradually turned brighter within the first $16 \mathrm{~s}$ which marked the progress to maximal resonance of PMBs, and while during 18$24 \mathrm{~s}$, the signal intensity slightly weakened due to the existence of a stable cavitation. Afterwards, the occurrence of an inertial cavitation was activated with the SI fading off from $26 \mathrm{~s}$ to $44 \mathrm{~s}$.

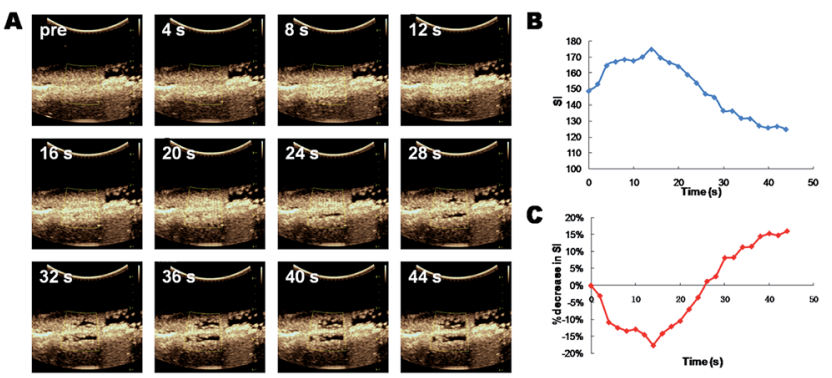

Fig. 3 The US images at varied pulse duration time (A) and the evaluation of cavitation effects with the analysis of signal intensity (B) and \% decrease (C) by using a convex transducer S1-8C. (US frequency: 3.3 $\mathrm{MHz}$, acoustical power: $60 \%$, US images recorded through a duration time of $44 \mathrm{~s}$, yellow line-confined area indicates ROI). 
These stages have been proved by the real-time US images, SI and \%decrease curves, and with these evidences, bubble resonance, stable and inertial cavitations could be readily differentiated. This provides us a visualized pathway to directly monitor the occurrence and progress of stable or inertial cavitations at the presence of PMBs. And more importantly in terms of theranostic applications, these findings are valuable to use PMBs for US imaging (at the time point of approximately $16 \mathrm{~s}$ with strongest signal due to the maximal resonance of PMBs), drug transportation (i.e. stable cavitation, before $24 \mathrm{~s}$ with drug loaded and delivered stably and visibly) and US-triggered stimuli-responsive release (i.e. inertial cavitation, during 26$44 \mathrm{~s}$ with PMBs collapse and subsequently drug deposition) in a live US image-guided manner.

\subsection{Comparison of cavitation effects at the presence of PMBs vs. Sonovue and XRX under optimized conditions}

To further investigate the performance of PMBs and realize their clinical translation and theranostic applications, it is of utmost importance to compare the cavitation behaviors of PMBs with commercial US contrast agents. Therefore, Sonovue and Xueruixin (XRX) as the representative contrast agents composed of lipids and proteins respectively were selected in our studies (Fig. 4A and B). ${ }^{35} 0.5 \mathrm{~mL}$ of MBs (PMBs, Sonovue, XRX) with a defined concentration of $1 \times 10^{9}$ MBs per $\mathrm{mL}$ were injected into deionized water for US imaging and cavitations at a clinically used frequency of $3.0 \mathrm{MHz}$ and $5.0 \mathrm{MHz}$, respectively. ${ }^{36}$ Based on previously optimized conditions, three different acoustical powers $(10 \%, 30 \%, 60 \%$ for $3.0 \mathrm{MHz}$, and $10 \%, 40 \%, 80 \%$ for $5.0 \mathrm{MHz}$ ) evoking total, partial and noninertial cavitation were selected. ROI was chosen where the MBs were uniformly dispersed.

As demonstrated in Fig. 4C and 5A, \%decrease in gray-scale for PMBs were almost equal to Sonovue and XRX, indicating

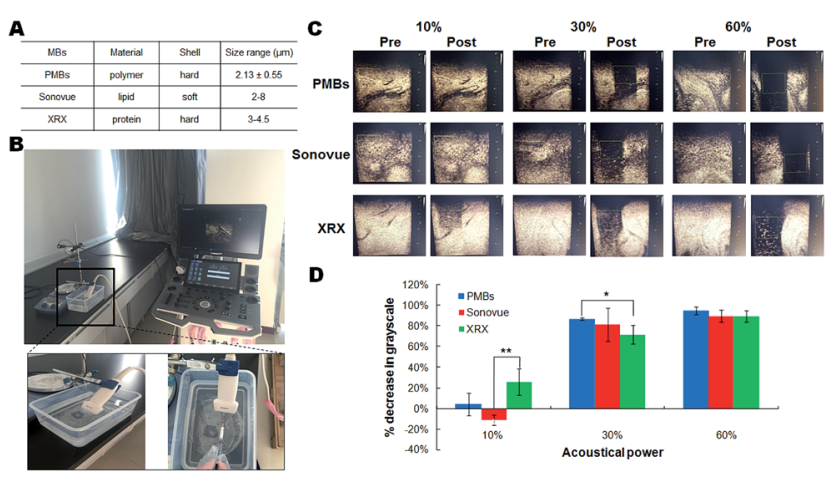

Fig. 4 Evaluation of cavitation behavior at the presence of PMBs, Sonovue and XRX. (A) Description of PMBs, Sonovue and XRX as representative microbubbles with distinct shell materials. (B) The apparatus for evaluating the cavitation behavior under optimized conditions. (C) US images before and after a single pulse for PMBs, Sonuvue and XRX at a frequency of $3.0 \mathrm{MHz}$ with defined acoustical powers of $10 \%, 30 \%$ and $60 \%$ (yellow line-confined area indicates ROI). (D) Significance analysis of \%decrease in gray-scale of US images. Values represent Mean $\pm \mathrm{SD}(n=3) . *$ and $* *$ indicate $p<0.05, p<0.01$ respectively with significant difference.

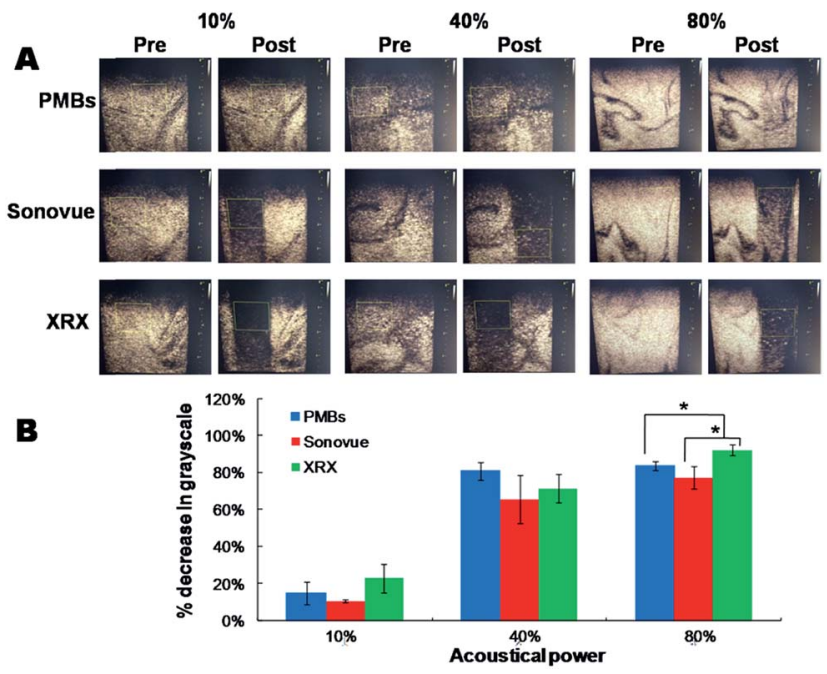

Fig. 5 Evaluation of cavitation behavior at the presence of PMBs, Sonovue and XRX at a frequency of $5.0 \mathrm{MHz}$ with defined acoustical powers of $10 \%, 40 \%$ and $80 \%$. (A) US images before and after a single pulse for PMBs, Sonuvue and XRX (yellow line-confined area indicates ROI). (B) Significance analysis of \%decrease in gray-scale of US images. Values represent Mean $\pm \mathrm{SD}(n=3)$. * indicates $p<0.05$ with significant difference.

that PMBs had excellent contrast performance as the commercial contrast agents. However, only obscure \%decrease in grayscale was observed for PMBs, Sonovue and XRX at an acoustical power of $10 \%$ at the frequency of either $3.0 \mathrm{MHz}$ or $5.0 \mathrm{MHz}$, which indicated no significant inertial cavitation under this low acoustical power. While the acoustical power was increased to $30 \%, 60 \%$ (in case of $3.0 \mathrm{MHz}$ ) and $40 \%, 80 \%$ (in case of 5.0 $\mathrm{MHz}$ ), significant inertial cavitations could be detected and PMBs displayed slightly better or comparable cavitation effects with Sonovue and XRX (Fig. 4D and 5B). As for $3.0 \mathrm{MHz}$ US irradiation (acoustical power 30\%), there was a significant difference of \%decrease in gray-scale between PMBs and XRX ( $p$ $<0.05$, Fig. 4D) that implied PMBs were more destructible than $\mathrm{XRX}$ under this condition. While in regard to 5.0 $\mathrm{MHz}$ US irradiation with higher acoustical power of $80 \%$, XRX showed a significantly better inertial cavitation performance than PMBs and Sonovue (Fig. 5B). In consequence, the as-synthesized polymeric microbubbles of PMBs not only have comparable contrast enhancement to Sonovue and XRX, but also are promising drug delivery agents with acceptable drug loading capacity when utilized to US-triggered cavitations as an efficacious strategy to serve the theranostic medicine.

\section{Conclusions}

In summary, the hard-shelled polymeric microbubbles (PMBs) have been synthesized via a one-step in situ polymerization, and characterizations proved them with narrow size distribution and good stability. As multifunctional agents for US imaging and cavitations, varied parameters including US frequency, acoustical powers and pulse duration time were screened to afford the optimized cavitation conditions. Meanwhile, it was 
notable to observe that by use of PMBs, a US-triggered progress of imaging, stable and inertial cavitations could be easily discriminated, and this endows a visualizable pathway to utilize PMBs as both US contrast agents and payload carriers for differentiating imaging, stimuli-responsive cavitation, drug transportation and release at each stage. To further investigate their availability, commercial US contrast agents of Sonovue and XRX with lipid and protein shell materials respectively were performed in comparison with the cavitation behavior of PMBs, and it was found that PMBs exhibited comparable competency as multifunctional US imaging and cavitation agents. It can be expected that with ligand conjugation on PMBs, specific biomarker-targeted capability will pave the way to diseaseoriented theranostics in association with US-triggered cavitation strategy. This will dramatically expand their biomedical application scope of polymeric microbubbles, and therefore provide accessibility to image-guided precision medicine.

\section{Acknowledgements}

This work was financially supported by the National Natural Science Foundation of China (21575106), the Scientific Research Foundation for Returned Scholars, Ministry of Education of China, Zhejiang Qianjiang Talents Program and Wenzhou Government's Start-up Fund. We authors are grateful to VINNO China for their generous technical assistance.

\section{Notes and references}

1 A. L. Klibanov and J. A. Hossack, Invest. Radiol., 2015, 50, 657-670.

2 H. Zhang, E. S. Ingham, M. K. J. Gagnon, L. M. Mahakian, J. Liu, J. L. Foiret, J. K. Willmann and K. W. Ferrara, Biomaterials, 2017, 118, 63-73.

3 I. De Cock, G. Lajoinie, M. Versluis, S. C. De Smedt and I. Lentacker, Biomaterials, 2016, 83, 294-307.

4 G. Dimcevski, S. Kotopoulis, T. Bjanes, D. Hoem, J. Schjott, B. T. Gjertsen, M. Biermann, A. Molven, H. Sorbye, E. McCormack, M. Postema and O. H. Gilja, J. Controlled Release, 2016, 243, 172-181.

5 B. Chertok, R. Langer and D. G. Anderson, ACS Nano, 2016, 10, 7267-7278.

6 F. Cavalieri, L. Micheli, S. Kaliappan, B. M. Teo, M. Zhou, G. Palleschi and M. Ashokkumar, ACS Appl. Mater. Interfaces, 2013, 5, 464-471.

7 T. Boissenot, A. Bordat, E. Fattal and N. Tsapis, J. Controlled Release, 2016, 241, 144-163.

8 A. Bouakaz, A. Zeghimi and A. A. Doinikov, Adv. Exp. Med. Biol., 2016, 880, 175-189.

9 J. Castle and S. B. Feinstein, Adv. Exp. Med. Biol., 2016, 880, 331-338.

10 F. Kiessling, J. Huppert and M. Palmowski, Curr. Med. Chem., 2009, 16, 627-642.

11 M. Poehlmann, D. Grishenkov, S. V. Kothapalli, J. Harmark, H. Hebert, A. Philipp, R. Hoeller, M. Seuss, C. Kuttner,
S. Margheritelli, G. Paradossi and A. Fery, Soft Matter, 2014, 10, 214-226.

12 I. Lentacker, I. De Cock, R. Deckers, S. C. De Smedt and C. T. Moonen, Adv. Drug Delivery Rev., 2014, 72, 49-64.

13 S. Hernot and A. L. Klibanov, Adv. Drug Delivery Rev., 2008, 60, 1153-1166.

14 K. Ferrara, R. Pollard and M. Borden, Annu. Rev. Biomed. Eng., 2007, 9, 415-447.

15 M. Postema and G. Schmitz, Ultrason. Sonochem., 2007, 14, 438-444.

16 F. Kiessling, S. Fokong, J. Bzyl, W. Lederle, M. Palmowski and T. Lammers, Adv. Drug Delivery Rev., 2014, 72, 15-27.

17 S. Fokong, M. Siepmann, Z. Liu, G. Schmitz, F. Kiessling and J. Gaetjens, Ultrasound Med. Biol., 2011, 37, 1622-1634.

18 Z. Liu, T. Lammers, J. Ehling, S. Fokong, J. Bornemann, F. Kiessling and J. Gaetjens, Biomaterials, 2011, 32, 61556163.

19 Z. Liu, P. Koczera, D. Doleschel, F. Kiessling and J. Gaetjens, Chem. Commun., 2012, 48, 5142-5144.

20 Z. Liu, C. Shi, Y. Li, Y. Song and Q. Xu, RSC Adv., 2016, 6, 32710-32714.

21 Z. Gao, A. M. Kennedy, D. A. Christensen and N. Y. Rapoport, Ultrasonics, 2008, 48, 260-270.

22 S. Mitragotri, Nat. Rev. Drug Discovery, 2005, 4, 255-260.

23 R. Bekeredjian, P. A. Grayburn and R. V. Shohet, J. Am. Coll. Cardiol., 2005, 45, 329-335.

24 P. A. Dayton, K. E. Morgan, A. L. Klibanov, G. H. Brandenburger and K. W. Ferrara, IEEE Trans. Ultrason. Eng., 1999, 46, 220-232.

25 C. M. Newman and T. Bettinger, Gene Ther., 2007, 14, 465475.

26 F. Forsberg, W. T. Shi, C. R. Merritt, Q. Dai, M. Solcova and B. B. Goldberg, J. Ultrasound. Med., 2005, 24, 443-450.

27 F. Forsberg, D. A. Merton and B. B. Goldberg, J. Ultrasound. Med., 2006, 25, 143-144.

28 S. L. Cibull, G. R. Harris and D. M. Nell, J. Ultrasound. Med., 2013, 32, 1921-1932.

29 J. B. Fowlkes and L. A. Crum, J. Acoust. Soc. Am., 1988, 83, 2190-2201.

30 C. C. Church, Ultrasound Med. Biol., 2003, 29, S56.

31 C. A. Macdonald, V. Sboros, J. Gomatam, S. D. Pye, C. M. Moran and W. Norman McDicken, Ultrasonics, 2004, 43, 113-122.

32 N. de Jong, A. Bouakaz and P. Frinking, Echocardiography, 2002, 19, 229-240.

33 J. Sijl, H. J. Vos, T. Rozendal, N. de, J. D. Lohse and M. Versluis, J. Acoust. Soc. Am., 2011, 130, 3271-3281.

34 M. J. Hsu, M. Eghtedari, A. P. Goodwin, D. J. Hall, R. F. Mattrey and S. C. Esener, J. Biomed. Opt., 2011, 16, 067002.

35 M. Schneider, M. Arditi, M. B. Barrau, J. Brochot, A. Broillet, R. Ventrone and F. Yan, Invest. Radiol., 1995, 30, 451-457.

36 S. L. Cibull, G. R. Harris and D. M. Nell, J. Ultrasound. Med., 2013, 32, 1921-1932. 